\title{
An implantable cardioverter defibrillator but not amiodarone reduced risk of death in congestive heart failure
}

Bardy GH, Lee KL, Mark DB, et al. Amiodarone or an implantable cardioverter-defibrillator for congestive heart failure. N Engl J Med 2005;352:225-37.

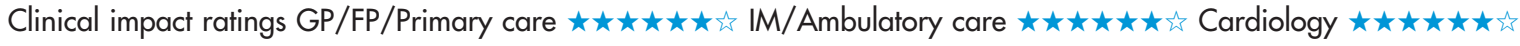

In patients with congestive heart failure (CHF), does amiodarone or an implantable cardioverter defibrillator (ICD) reduce all cause mortality more than placebo?

\section{METHODS}

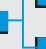

Design: randomised placebo controlled trial (Sudden Cardiac Death in Heart Failure Trial [SCD-HeFT]).

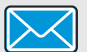

Allocation: concealed.*

Blinding: blinded (clinicians and patients).*

Follow-up period: median 46 months.

Setting: 145 centres in Canada and the US.

Patients: 2521 patients who were $\geqslant 18$ years of age (median age 60 y, 77\% men) with New York Heart Association (NYHA) class II or III chronic, stable CHF from ischaemic causes (left ventricular systolic dysfunction [LVSD] with marked coronary artery stenosis or documented myocardial infarction) or nonischaemic causes (LVSD without marked stenosis); and an LV ejection fraction $<35 \%$.

D Intervention: amiodarone (loading dose of $800 \mathrm{mg}$ daily for $1 \mathrm{wk}, 400 \mathrm{mg}$ daily for $3 \mathrm{wks}$, then a weight dependent daily dose [200-400 mg]) ( $\mathrm{n}=845)$; a single chamber ICD device programmed to shock only mode (model 7223 , Medtronic) with a detection rate of $\geqslant 187$ beats/minute for only rapid, sustained ventricular tachycardia or ventricular fibrillation $(\mathrm{n}=829)$; or placebo ( $n=847)$.

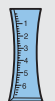

Outcomes: all cause mortality and adverse effects.

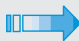

Patient follow up: $100 \%$ (intention to treat analysis)

*See glossary.

Amiodarone (Amio) or a conservatively programmed, shock only implantable cardioverter defibrillator (ICD) v placebo for congestive heart failure at median 46 months*

\begin{tabular}{llllll}
\hline Outcome & Amio & ICD & Placebo & RRR (95\% CI) & NNT (CI) \\
\hline $\begin{array}{l}\text { All cause } \\
\text { mortality }\end{array}$ & $9.5 \%$ & $7.2 \%$ & $9.7 \%$ & $1.6 \%(-16$ to 17$)$ & NS \\
\hline
\end{tabular}

${ }^{*} \mathrm{NS}=$ not significant. Other abbreviations defined in glossary; RRR, $\mathrm{NNT}$, and $\mathrm{Cl}$ calculated from data in article.

For correspondence: $\operatorname{Dr}$ G H Bardy, Seattle Institute for Cardiac Research, Seattle, WA, USA. gbardy@sicr.org

Sources of funding: National Institutes of Health; Medtronic; Wyeth-Ayerst Laboratories; Knoll Pharmaceuticals.

\section{MAIN RESULTS}

$70 \%$ of patients had NYHA class II CHF, and $30 \%$ had class III CHF. 188 patients $(11 \%)$ from the amiodarone and placebo groups crossed over to some form of ICD therapy. 259 patients in the ICD group $(31 \%)$ received shocks from their device for any cause. Fewer patients who received ICD died from any cause than did those who received placebo (table). Amiodarone and placebo groups did not differ for all cause mortality (table). Compared with placebo, amiodarone increased tremor $(\mathrm{P}=0.02)$ and hypothyroidism $(\mathrm{P}<0.001)$. In patients with NYHA class III CHF, ICD and placebo groups did not differ for mortality (hazard ratio [HR] 1.16, 97.5\% CI 0.84 to 1.61 ); amiodarone increased mortality more than placebo (HR 1.44, CI 1.05 to 1.97). In patients with NYHA class II CHF, amiodarone and placebo groups did not differ for mortality (HR 0.85, CI 0.65 to 1.11); ICD decreased mortality more than placebo (HR 0.54 , CI 0.40 to 0.74 ).

\section{CONCLUSIONS}

In patients with congestive heart failure ( $\mathrm{CHF}$ ), a conservatively programmed, shock only implantable cardioverter defibrillator reduced all cause mortality. Compared with placebo, ICD reduced mortality in New York Heart Association (NYHA) class II but not class III CHF. Amiodarone had no effect in NYHA class II CHF, and increased mortality in NYHA class III CHF.

Abstract and commentary also appear in ACP Journal Club.

\section{Commentary}

The SCD-HeFT trial provides evidence for prophylactic use of ICDs. The historical importance of SCD-HeFT and its effect on clinical practice must account for the trial's immediate influence on Medicare guidelines. SCD-HeFT reinforced earlier studies on the use of prophylactic ICDs in ischaemic and non-ischaemic cardiomyopathy ${ }^{1-2}$ The inclusion and exclusion criteria of SCD-HeFT were rapidly incorporated into Medicare's reimbursement plan. According to the Centres for Medicare and Medicaid (CMS), these changes in ICD eligibility will increase the number of potential ICD patients by one third, to a total of $500000 .^{3}$ CMS also mandated that the single chamber, less expensive ICD that was used in SCD-HeFT be used unless otherwise justified. Despite using single chamber ICDs, the diagnosis-related group cost of US $\$ 25000$ to $\$ 30$ 000 per ICD is still a concern.

SCD-HeFT also included an amiodarone arm, which showed no benefit in the primary prevention of sudden cardiac death (SCD). The large size of the SCD-HeFT trial clarifies questions asked by previous smaller and sometimes conflicting studies of amiodarone for SCD prophylaxis. This lack of survival benefit will probably end almost a decade of research in SCD prevention with amiodarone. Although the SCD-HeFT trial provided many answers in SCD prevention, the methods used to identify patients with the highest risk are still primitive. Further trials will help determine whether the specific exclusions of SCD-HeFT have biological meaning and justification as implantation and reimbursement criteria.

Andrew Corsello, MD Maine Medical Centre Portland, Maine, USA

1 Moss AJ, Zareba W, Hall WJ, et al. N Engl J Med 2002;346:877-83.

2 Kadish A, Dyer A, Daubert JP, et al. N Engl J Med 2004;350:2151-8.

3 Centers for Medicare and Medicaid Services. Medicare news.

www.cms.hhs.gov/media/press/release. asp? Counter $=1331$ (accessed 6 Jun, 2005). 